\title{
A mutation in NOTCH1 ligand binding region detected in patients with oral squamous cell carcinoma reduces NOTCH1 oncogenic effect
}

\author{
MASAHIRO UCHIBORI $^{1,2}$, KEN-ICHI AOYAMA ${ }^{1,2}$, YOSHIHIDE OTA ${ }^{1}$, \\ KAGEMASA KAJIWARA $^{2}$, MASAFUMI TANAKA ${ }^{2}$ and MINORU KIMURA ${ }^{2}$ \\ Departments of ${ }^{1}$ Oral and Maxillofacial Surgery, ${ }^{2}$ Molecular Life Science, \\ Tokai University School of Medicine, Isehara, Kanagawa 259-1193, Japan
}

Received February 10, 2017; Accepted July 11, 2017

DOI: $10.3892 /$ or.2017.5870

\begin{abstract}
NOTCH1 is known as an oncogenic or tumor suppressive gene in solid cancer. NOTCH1 mutations in oral squamous cell carcinoma (OSCC) frequently occur near the ligand-binding region. These mutations change the domain structure of this protein and affect the ligand binding activity. When NOTCH1 is activated by ligand binding, NOTCH1 intracellular domain (NICD) is cleaved from the cell membrane. This study investigated the functional change induced by a NOTCH1 mutation detected in OSCC clinical samples using stable transformant analysis. HEK293 cell lines expressing NOTCH1 wild-type (WT cells) or p.A465T NOTCH1 (A465T cells) were established. NOTCH1 expression was analyzed by flow cytometry, western blotting, and immunofluorescence using an anti-human NOTCH1 antibody. mRNA expression levels in WT and A465T cells were determined by quantitative real-time PCR (qPCR). Cell proliferation was analyzed by using cell growth assays and a xenograft tumor assay. Flow cytometry indicated that NOTCH1 expression on the cell membrane was lower in A465T cells than that in WT cells. NOTCH1 and NICD were both detected by western blot in WT and A465T cells. The immunofluorescence signal for NICD was detected in the nucleus of WT cells, while it was localized mainly in the cytoplasm of A465T cells. HES1 and HEY1 mRNA expression levels were lower in A465T than in WT cells. The cell growth of WT cells was significantly higher than that of HEK293 cells (3-fold, $\mathrm{P}<0.01$ ), while that of A465T cells was significantly lower than that of HEK 293 cells $(37 \%$, $\mathrm{P}<0.01)$. In a xenograft model, the tumor cell implantation rate of WT cells was $80 \%$, while that of A465T cells was $0 \%$.
\end{abstract}

Correspondence to: Professor Minoru Kimura, Department of Molecular Life Science, Tokai University School of Medicine, 143 Shimokasuya, Isehara, Kanagawa 259-1193, Japan

E-mail: kimura@is.icc.u-tokai.ac.jp

Key words: oral cancer, head and neck cancer, Japanese oral squamous cell carcinoma, NOTCH1 mutation, NOTCH1 ligand-binding region
This study indicates that NOTCHI acts as an oncogene and that the NOTCHI mutation (p.A465T) in the ligand-binding region causes the loss of tumorigenicity by downregulating the NOTCH1 pathway.

\section{Introduction}

Oral cancer is a rare cancer that represents $1 \%$ of solid cancers. Squamous cell carcinoma (SCC) represents $90 \%$ of oral cancer. The incidence rate depends on populations because oral squamous cell carcinoma (OSCC) is associated with tobacco use and alcohol consumption $(1,2)$. There is no specific biomarker and the standard treatment for OSCC is dissection (3). Although several cancer genes are known as drivers in head and neck squamous cell carcinoma (HNSCC), there is no specific therapeutic molecule (4-6). Recent sequencing studies examined HNSCC, including OSCC occurring at a heterogeneous set of anatomical sites (1,7-9). NOTCH1 mutations are frequently detected in OSCC in addition to TP53, CDKN2A, PIK3CA, and HRAS mutations. These mutations, including NOTCH1 mutations, are known in solid cancers $(1,7,8,10)$. Studies on Caucasian populations indicate that $\mathrm{NOTCH} 1$ mutations might be associated with worse prognosis, but studies on Asian populations indicate an oncogenic role for NOTCHI (1).

$\mathrm{NOTCH}$ family includes four members (termed NOTCH1-4), which are type 1 transmembrane receptors. NOTCH1 affects proliferation, differentiation, and apoptosis of diverse cell types in various organisms (11). NOTCH proteins are composed of an extracellular domain (NECD) and an intracellular domain (NICD). The NECD contains epidermal growth factor repeats (EGFr) 1-36 and EGFr 12 is the ligandbinding domain. When EGFr 12 binds to its ligands, jagged and delta family proteins, the NOTCH receptor is cleaved. After cleavage of the NOTCH receptor, the NICD is released from the cell membrane and it migrates to the nucleus. The NICD interacts with RBP-J, which is a DNA-binding protein. Finally, these complexes activate HES or HEY family of transcription genes, thereby inducing downstream factors (11). NOTCH signaling presents an oncogenic or tumor suppressive effect, owing to crosstalk with the EGF receptor (EGFR) pathway and 
the subsequent activation of the PI3K-AKT pathway, KRAS, CCND1, and matrix metalloproteinase 9 (MMP9) (11).

NOTCH1 is a well-known oncogene in blood cancer; $50 \%$ of patients with T-cells acute lymphoblastic leukemia (T-ALL) present NOTCH1 mutations (12). However, the mutation spectrum of NOTCHI in OSCC is different from that of T-ALL. Therefore, the function of NOTCH1 in solid cancer is unclear. Recent studies suggested that NOTCH1 may play an oncogenic or tumor suppressive role in solid cancer because the NOTCH1 pathway regulates various oncogenes and tumor suppressor genes such as c-Myc, PI3K, RAS family, EGFR, PTEN, and $T P 53$. For example, both overexpression and downregulation of NOTCH and ligands have been implicated in several human cancers, including OSCC, in clinical studies $(11,13,14)$. For HNSCC, there are some studies using cancer cell lines, but there is no report using clinical samples. Thus, the function of NOTCH1 in tumors is unsettled (15). We previously reported that NOTCH1 mutations in Japanese patients with OSCC frequently occur near the ligand-binding region $(9.5 \%, 8$ of 84 patients with OSCC). These mutations are thought to induce a conformational change in NOTCH1 and its downregulation (16).

At present, there is no report on the function of NOTCH1 mutations detected in OSCC clinical samples. In this study, we examined the expression and the functional change of a NOTCH1 mutant (p.A465T) detected in OSCC clinical samples using stably transformed HEK293 cells.

\section{Materials and methods}

Construction of expression vectors. The vectors contained the EBV promoter-cDNA of NOTCH1 gene [wild-type (WT) NOTCH1, NOTCH1 mutant [G1393A (p.A465T)], or empty vector (MOCK)]-IRES-anti-hygromycin. The G1393A was introduced in NOTCH1 (NM_017617) human ORF cDNA Clone (Origene Technologies, Inc., Rockville, MD, USA) by in vitro mutagenesis. The cDNA was cloned into the pCEP4 vector (Invitrogen, Carlsbad, CA, USA) between the SalI and NruI restriction sites as previously described (17).

Cell culture and transfection. HEK293 cells and transformants were cultured in Dulbecco's modified Eagle's medium with $10 \%$ fetal bovine serum under $5 \% \mathrm{CO}_{2}$ at $37^{\circ} \mathrm{C}$. HEK 293 cells were transfected using the WT NOTCH1 and p.A465T NOTCH1 mutant expression vectors with X-tremeGENE HP Reagent (Roche, Basel, Switzerland) in a 6-well plate, and cells were cultured with hygromycin $(400 \mu \mathrm{g} / \mathrm{ml})$ over 21 days. Stable transformants were used for all assays.

Flow cytometry. Cells were collected by trypsinization followed by centrifugation at $500 \mu \mathrm{g}$ for $5 \mathrm{~min}$. Cell pellets were then resuspended in $100 \mu \mathrm{l}$ of phosphate buffered saline (PBS, Life Technologies, Carlsbad, CA, USA). The following antibodies were used for fluorescence-activated cell sorting (FACS) analysis: PE anti-human Notch1 antibody (MHN1-519) (BioLegend, San Diego, CA, USA) and PE mouse IgG1 $\kappa$ Isotype control (BioLegend; negative control). After incubation of the cells with $1 \mu \mathrm{l}$ of antibody for $20 \mathrm{~min}$ and washing in PBS, cells were incubated at $4^{\circ} \mathrm{C}$ in the dark for $1 \mathrm{~h}$. They were then analyzed using a FACS Aria cell sorter (BD Biosciences, San Jose, CA, USA).
Western blotting. Cell extracts were prepared using an ultrasonic disrupter, and protein concentration was determined by DC protein assay (Bio-Rad, Hercules, CA, USA). T Protein (25 $\mu \mathrm{g})$ were loaded on $7.5 \%$ SDS/PAGE pre-casted gels (e-PAGEL, ATTO, Tokyo, Japan) and transferred to polyvinylidene fluoride membranes (Millipore, Billerica, MA, USA). The primary antibody used was the NOTCH1 antibody (1:1,000, \#3608, Cell Signaling Technology, Danvers, MA, USA), which recognizes the C-terminal of NOTCH1, and the secondary antibody was the peroxidase-conjugated rabbit anti-rabbit IgG antibody (1:2,000, \#7074, Cell Signaling Technology). Rabbit anti-GAPDH antibody (1:3,000, G9545, Sigma-Aldrich, St. Louis, MO, USA) was used for standardization. Detection was carried out by chemiluminescence using Western Lightning Ultra (Perkin-Elmer, Waltham, MA, USA).

Immunofluorescence imaging. WT and A465T cells cultured on glass slides were washed three times with PBS, fixed in $4 \%$ paraformaldehyde (PFA) for $15 \mathrm{~min}$ at room temperature, washed 3 times with PBS, and incubated with $10 \%$ goat serum (Rockland Immunochemicals, Inc., Pottstown, PA, USA) for $10 \mathrm{~min}$ at room temperature. After washing, the cells were incubated with a rabbit monoclonal anti-NOTCH1 antibody (1:100, \#3608, Cell Signaling Technology) diluted in $0.05 \%$ Triton X100/1\% BSA/0.01 M PBS at $4{ }^{\circ} \mathrm{C}$ for $16 \mathrm{~h}$. After being washed with PBS, the cells were incubated with Alexa Fluor $^{\circledR} 488 \mathrm{~F}\left(\mathrm{ab}^{\prime}\right)_{2}$ fragment goat anti-rabbit $\operatorname{IgG}(\mathrm{H}+\mathrm{L})(1: 200$, Molecular Probes, Eugene, OR, USA) at room temperature for $1 \mathrm{~h}$. Cells were washed 3 times, and nuclei were stained with 4',6-diamidino-2-phenylindole, dihydrochloride (DAPI) (Sigma-Aldrich). Cell fluorescence was analyzed by laser scanning confocal microscopy (LSM700; Carl Zeiss, Jena, Germany).

Quantitative real-time PCR. Total RNA was extracted from WT cells, A465T cells, and MOCK cells by using TRIzol reagent (Invitrogen). RNA was reverse-transcribed to cDNA. Primers were as follows: HES1, forward 5'-gaagcacctccggaa cct-3', reverse 5'-gtcacctcttcatgcactc-3'; HEY1, forward 5'-cata cggcaggagggaaag-3', reverse 5'-gcatctagtccttcaatgatgct-3'. These primers were designed by using primer3plus (http:// www.bioinformatics.nl/cgi-bin/primer3plus/primer3plus.cgi). Quantitative real-time PCR (qPCR) amplification was performed using Fast SYBR-Green Master Mix (Thermo Scientific, Waltham, MA, USA) according to the manufacturer's instructions. $\beta$-actin was used as the housekeeping gene.

Cell growth assay. The Cell Counting Kit-8 (CCK-8) (Dojindo Laboratories, Kumamoto, Japan) was used to determine cell proliferation of WT, A465T, and MOCK cells. Two thousand five hundred cells per well were cultured in 96-well culture plates in Dulbecco's modified Eagle's medium with $10 \%$ fetal bovine serum for $96 \mathrm{~h}$. CCK-8 solution $(5 \mu \mathrm{l})$ were added to the cells for $2 \mathrm{~h}$ at $37^{\circ} \mathrm{C}$, and optical density (OD) was examined at a wavelength of $450 \mathrm{~nm}$ using a spectra $\mathrm{Max}^{\circledR} \mathrm{i} 3$ (Molecular Devices, Sunnyvale, CA, USA) according to the manufacturer's instructions.

Xenograft model. WT, A465T, and MOCK cells $\left(5 \times 10^{6}\right)$ were suspended in a 1:1 mixture of $200 \mu \mathrm{l}$ PBS and Matrigel 


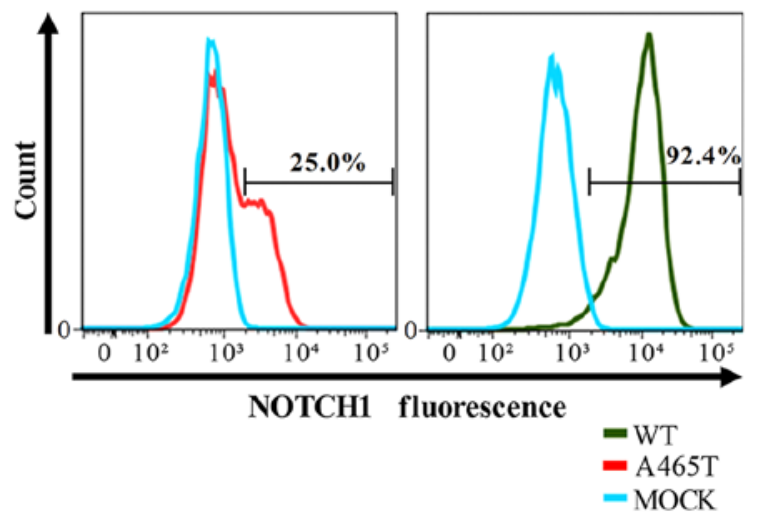

Figure 1. Flow cytometry analysis of WT and A465T cells. NOTCH1 expression was measured by assessing PE fluorescence. The number of NOTCH1 positive cells were 9240 cells $(92.4 \%)$ and 2500 cells $(25.0 \%)$ in WT and p.A465T transformants, respectively. MOCK cells were used as negative controls (10 NOTCH1 positive cells, $0.1 \%$ ).

(Corning, Tewksbury, MA, USA) and injected subcutaneously into the flank of five female BALB/C nude mice (5 weeks old) for each group (CLEA Japan, Tokyo, Japan). At 8 weeks after transplantation, we evaluated the cell implantation rate. All experiments were approved by the Animal Ethics Committee of Tokai University School of Medicine. These tumors were excised and preserved in $10 \%$ formalin. Hematoxylin and eosin (H\&E) staining was performed by using a standard technique. Immunohistochemical staining (IHC) was performed by using a rabbit monoclonal anti-NOTCH1 antibody (1:100, \#3608, Cell Signaling Technology).

Statistical analysis. Statistical analyses were performed by using the SPSS version 23 software (SPSS, Chicago, IL, USA). The data shown represent mean values \pm SD. The statistical analyses were performed using the Student's t-test, and $\mathrm{P}<0.01$ was considered to indicate a statistically significant difference.

\section{Results}

Flow cytometry. The percentage of NOTCH1 positive cells was $92.4 \%$ and $25.0 \%$ in WT and p.A465T transformants, respectively. MOCK cells were used as a negative control (NOTCH1 positive cells: $0.1 \%$ ). The histogram of A465T cells was diphasic, and the fluorescence intensity of positive A465T transformed cells was lower than that of WT transformed cells (Fig. 1). We verified the mRNA expression level of each transfected vector. The mRNA expression was standardized to that in MOCK cells. The NOTCH1 mRNA level was 1.78 (WT/MOCK) in WT cells and that of A465T cells was 0.48 (p.A465T/MOCK) consistent with the FACS analysis.

Western blotting. NOTCH1 and NICD were detected both in WT and A465T cells. NOTCH1 and NICD expression levels were lower in A465T cells than those in WT cells, but the NICD expression level, as compared to that of NOTCH1, was relatively higher in A465T cells than that in WT cells (Fig. 2).

Immunofluorescence imaging. Consistent with previous studies, NICD was detected in the nucleus in WT cells.

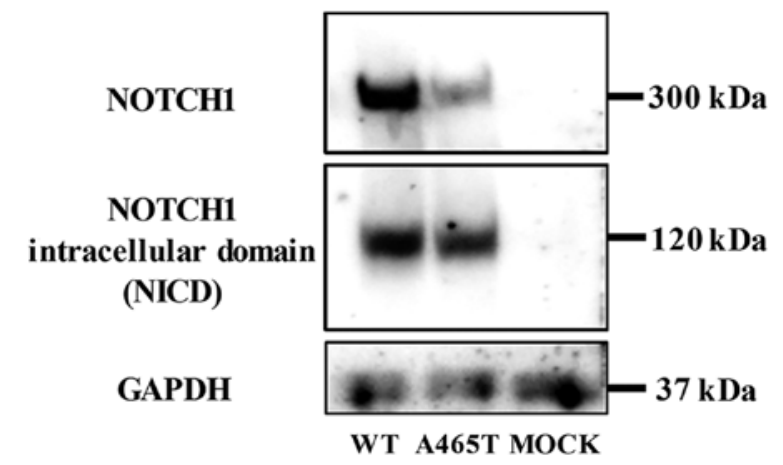

Figure 2. Western blot analysis of NOTCH1 and NICD expression in WT and A465T cells. MOCK cells were used as a control. GAPDH was used as an internal loading control.

However, we detected a strong NICD signal in the cytoplasm of A465T cells and no signal in the nucleus. This means that NICD was equally expressed in WT and A465T cells, but the localization of the NICD in A465T cells was different from that in WT cells (Fig. 3).

Quantitative real-time PCR. We measured HES1 and HEY1 mRNA expression level in WT and A465T by qPCR. mRNA expression was standardized to that in MOCK cells. The HES1 mRNA expression levels in WT and A465T cells were 3.55 and 0.38 , respectively (Fig. 4A). The HEY1 mRNA expression levels in WT and A465T cells were 5.15 and 079, respectively (Fig. 4B).

Cell growth. The cell growth rates of WT and A465T cells, measured as a ratio of the cell number using MOCK cells as a standard, were 313 and 37\%, respectively. The A465T cell number was significantly lower than the MOCK cell number $(\mathrm{P}<0.01)$, while the WT cell number was significantly higher than the MOCK cell number $(\mathrm{P}<0.01)$ (Fig. 5).

Xenograft model. WT, A465T, and MOCK cells were injected subcutaneously in the flanks of five mice per group. WT cell implantation was observed in the flank of four out of five mice (implantation rate: $80 \%$ ). MOCK cell implantation was detected in the flank of three out of five mice (implantation rate: $60 \%$ ). However, no implantation was observed in mice injected with A465T cells. Tumors generated from WT cells were obviously larger than those generated from MOCK cells, consistent with the cell growth assay results (Fig. 6A). We performed $H \& E$ staining on tumors generated from each NOTCH1 WT and MOCK cells in the flanks of nude mice at 8 weeks after implantation. Macroscopically, nude mice presented a well-circumscribed margin and slight fibrosis of the tumor masses. Cell density of WT cells was higher than that of MOCK cells. Immunohistochemical staining showed that all cells were NOTCH1 positive (Fig. 6B).

\section{Discussion}

NOTCH1 mutations are the most frequently detected mutations in HNSCC (7). These mutations are specifically detected in the vicinity of the ligand-binding region of NOTCH1 


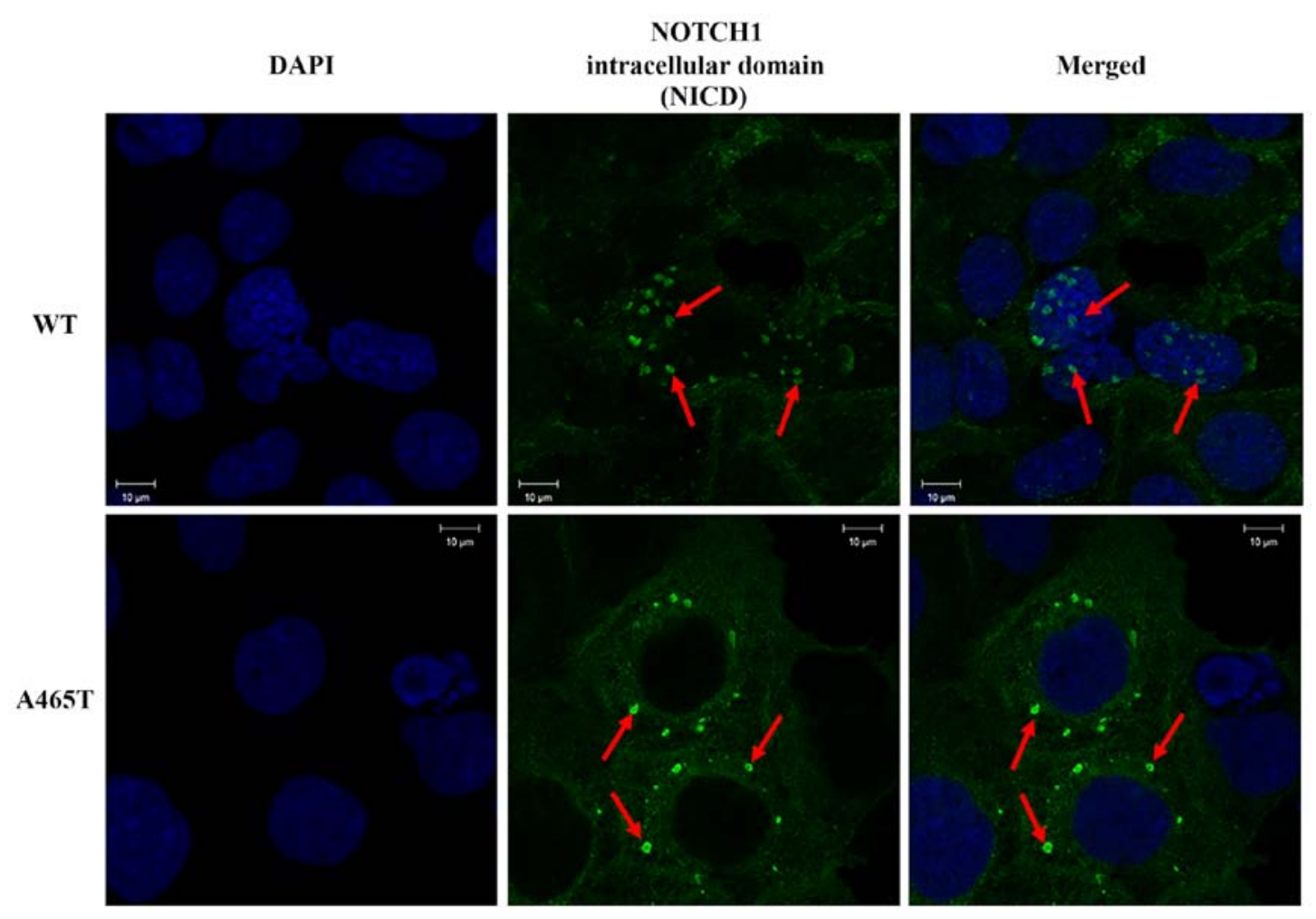

Figure 3. Immunofluorescence imaging of NICD in WT and A465T cells. Red arrows indicate NICD.

A

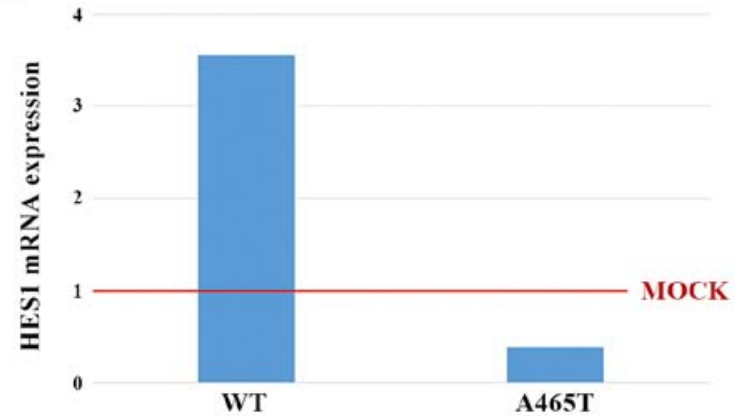

B

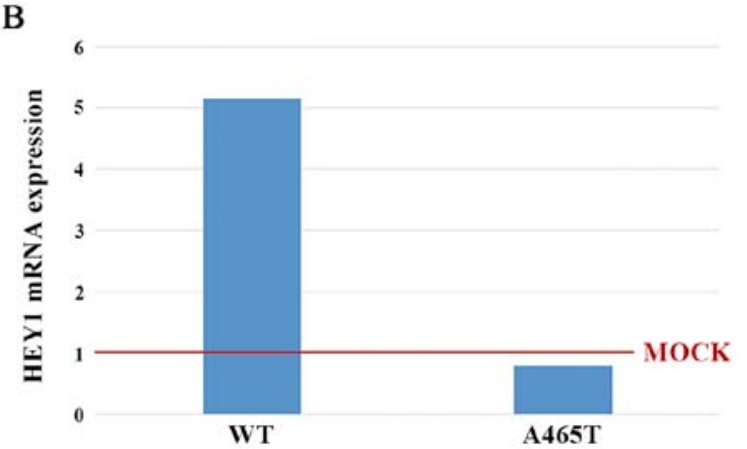

Figure 4. HES1 (A) and HEY1 (B) mRNA expression levels in WT, A465T, and MOCK cells assessed by quantitative real-time PCR. Values are normalized to $\beta$-actin. Error bars represent standard errors.

in patients with OSCC and HNSCC $(7,16,18)$. Among these mutations, the G1393A (p.A465T) mutant identified in two Japanese OSCC patients is located in EGFr12, and the other six mutations are located in EGFr10. A previous report showed that the G1393A (p.A465T) mutation leads to a conformational change of NOTCH1 ligand-binding domain by protein structure simulation (16). However, recent studies using cancer cell lines could not provide evidence about the functional role of NOTCH1 mutations detected in clinical samples $(15,19,20)$. Thus, to clarify the function of NOTCH1 in OSCC, we established A465T cells expressing the NOTCH1 A465T mutant and examined its characteristics.

Generally, the process of NOTCH1 activation requires some key steps. The first step is ligand binding, which requires NOTCH1 localization on the cell membrane. The second step is NOTCH1 cleavage between the NECD and NICD $(21,22)$. Finally, the translocation of NICD into the nucleus leads

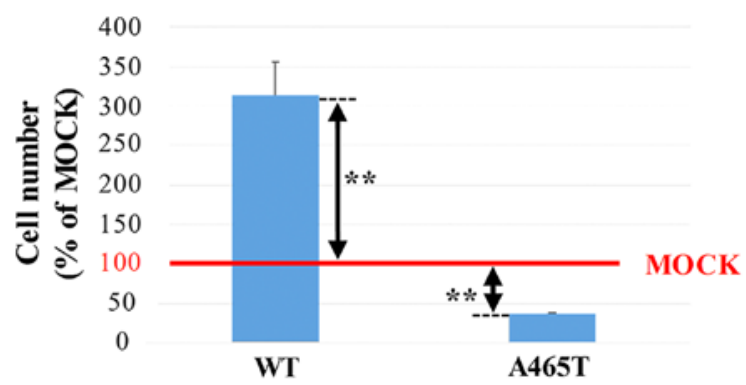

Figure 5. Cell growth assay using WT and A465T expressing cells. ${ }^{* *} \mathrm{P}<0.01$ as determined by Student's t-test. Error bars represent standard errors.

to the activation of NOTCH1 signaling (23). To examine the NOTCH1 activity between WT and A465T cells, we performed flow cytometry analysis to quantify the amounts 
A

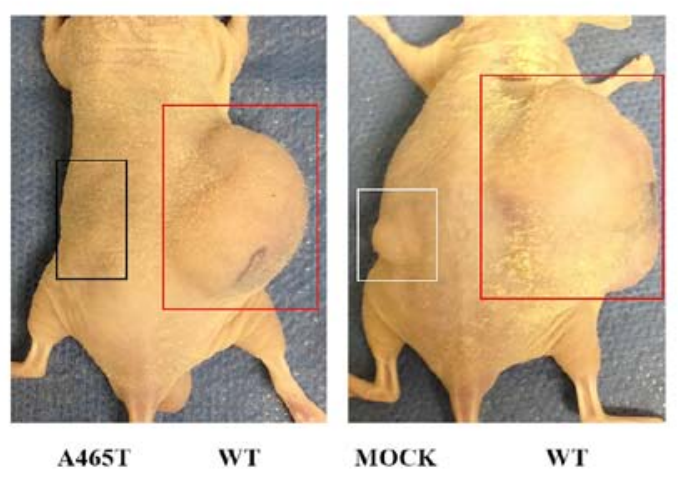

B

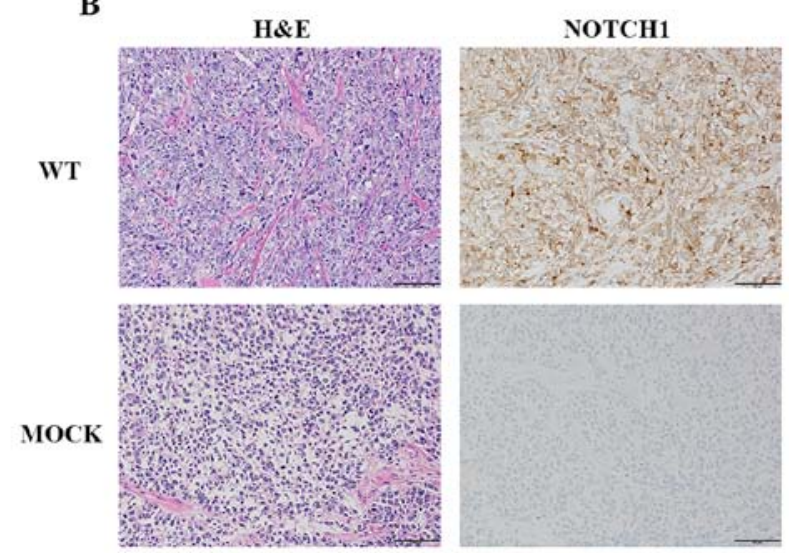

Figure 6. Xenograft mouse model. (A) Red, black, and white squares indicate the injection site and/or xenograft generated by injection of WT, A465T, or MOCK cells, respectively. (B) Xenograft tumor sections of WT and MOCK cells were stained with hematoxylin and eosin (H\&E) and with an antiNOTCH1 antibody.

of NOTCH1 localized on the cell surface and western blot analysis to evaluate NOTCH1 cleavage by detecting NICD and NOTCH1 (24). Although NOTCH1 A465T expression was downregulated on the cell surface as shown by flow cytometry analysis, both the cleaved NICD and full length NOTCH1 were detected in the A465T cells by western blot analysis. Consistent with our findings, a previous in silico report suggested that p.A465T mutation affects the conformation of NOTCH1 ligand binding region and downregulates NOTCH1 function (16). Thus, we hypothesized that NOTCH1 A465T would be downregulated and the NICD would not be localized in the nucleus. As mentioned above, NICD has to be cleaved from NOTCH1 in the cell membrane in order to migrate to the cytoplasm or nucleus. To examine whether the NICD from NOTCH1 A465T participates in the NOTCH1 signaling, we next analyzed NICD localization by immunofluorescence and confocal microscopy. The NICD in A465T mutant cells was localized in the cytoplasm instead of in the nucleus by immunofluorescence analysis. Furthermore, HES1 and HEY1 mRNA expression levels were decreased in A465T cells compared with that in MOCK cells. A recent report demonstrated that a decrease in NOTCH1 mRNA expression induces a decrease in HESI and HEY1 mRNA expression (25). Together with this study, our findings suggest that NOTCH1 activity declined in A465T cells.

O-fucosylation is an important factor in the maturation of NOTCH1 because the chaperone activity must be sufficient to support ligand binding (26). Furthermore, the disruption of a single EGFr can dominantly perturb NOTCH activation (27). In this study, although NOTCH1 A465T was cleaved, the NICD could not translocate to the nucleus. According to these reports, we considered that the conformational change in the NICD structure was associated with some modifications, including $\mathrm{O}$-fucosylation, or with a change in the structure of the EGFrs induced by p.A465T mutation, which lead to NOTCH1 downregulation.

We next performed cell growth assay and xenograft implantation to evaluate the effect of WT and A465T NOTCH1 on NOTCH1-related tumorigenesis. Cell growth assay showed that the proliferation of WT cells increased, while that of A465T cells decreased. The growth of NICD overexpressing cells increased and that of NOTCH1 knocked down cells decreased in vitro $(20,28)$. In addition, recent reports demonstrated that siRNA-mediated silencing of mRNA for both HEY1 and NOTCH1 causes lower levels of cell proliferation (29). In our study, HEYl expression levels were lower in A465T cells than in WT cells. The implantation rate of cells in a xenograft model represents the resistance of cell lines to immunosuppression and their adaptation to the host microenvironment (30). NOTCH1 regulates the formation of the microenvironment (31). Our xenograft model showed that no implantation was observed when A465T cells were injected to the mice, while WT expressing cells were implanted at a higher rate than MOCK cells. Thus, our cell growth assay and xenograft model indicated that WT NOTCH1 leads to increases tumorigenicity, while p.A465T mutation abolishes it. These data are consistent with our previous report that median disease-free survival of OSCC patients with NOTCH1 mutation in the vicinity of the ligand binding region is significantly longer than that of patients presenting with WT NOTCH1 (16). Together with previous evidence, these findings suggest that the p.A465T mutation mediates NOTCH1 downregulated tumorigenicity.

In this study, we expected a negative relationship between p.A465T mutation and NOTCH1 activity. However, our results did not directly demonstrate whether NOTCH1 activity was lost in A465T cells. Further experiments are required to determine whether p.A465T located in EGFr 12 leads to the instability of NOTCH1 within cell membrane. However, this mutation did not directly contribute to the defect in NICD migration as shown in Fig. 3. Secondly, since NOTCH signaling crosstalks with other pathways associated with tumorigenicity, other molecular factors might abolish NOTCH1 tumorigenicity. Third, ligand stimulation is followed by the cleavage step of NOTCH1. Thus, we expect that the p.A465T mutant could lead to alternative cleavage of NOTCH1, resulting in structural changes and/or unusual migration of NICD.

In conclusion, our findings suggest that NOTCH1 acts as an oncogene and that p.A465T NOTCH1 mutation in the ligand-binding region affect cell growth and/or tumorigenicity of OSCC. Furthermore, we consider that NOTCH1 mutations in the ligand-binding region could be good 
prognostic factors and the domain could be used as a new therapeutic molecule in OSCC. Further studies are warranted to elucidate the direct relationship between the p.A465T mutant and NOTCH1 activity.

\section{Acknowledgements}

We are grateful to the Support Center for Medical Research and Education, Tokai University, for technical support. This work was supported by Grants-in-Aid for Young scientists (B) (KAKENHI Grant Number 16K20614) from Japan Society of the Promotion of Science (JSPS), Grants-in-Aid for Scientific Research (C) (KAKENHI Grant Number 15K11302) from JSPS and 2015 Tokai University School of Medicine Research Aid. We thank Editage for language editing.

\section{References}

1. Chaturvedi AK, Anderson WF, Lortet-Tieulent J, Curado MP, Ferlay J, Franceschi S, Rosenberg PS, Bray F and Gillison ML: Worldwide trends in incidence rates for oral cavity and oropharyngeal cancers. J Clin Oncol 31: 4550-4559, 2013.

2. Warnakulasuriya S: Global epidemiology of oral and oropharyngeal cancer. Oral Oncol 45: 309-316, 2009.

3. Pfister DG, Spencer S, Brizel DM, Burtness B, Busse PM, Caudell JJ, Cmelak AJ, Colevas AD, Dunphy F, Eisele DW, et al: Head and Neck Cancers, Version 1.2015. J Natl Compr Canc Netw 13: 847-856, 2015.

4. Chi LM, Lee CW, Chang KP, Hao SP, Lee HM, Liang Y, Hsueh C, Yu CJ, Lee IN, Chang YJ, et al: Enhanced interferon signaling pathway in oral cancer revealed by quantitative proteome analysis of microdissected specimens using 16O/18O labeling and integrated two-dimensional LC-ESI-MALDI tandem MS. Mol Cell Proteomics 8: 1453-1474, 2009.

5. Mazumdar A, Henderson YC, El-Naggar AK, Sen S and Clayman GL: Aurora kinase A inhibition and paclitaxel as targeted combination therapy for head and neck squamous cell carcinoma. Head Neck 31: 625-634, 2009.

6. Colella S, Richards KL, Bachinski LL, Baggerly KA, Tsavachidis S, Lang JC, Schuller DE and Krahe R: Molecular signatures of metastasis in head and neck cancer. Head Neck 30 : 1273-1283, 2008.

7. Stransky N, Egloff AM, Tward AD, Kostic AD, Cibulskis K, Sivachenko A, Kryukov GV, Lawrence MS, Sougnez C, McKenna A, et al: The mutational landscape of head and neck squamous cell carcinoma. Science 333: 1157-1160, 2011.

8. Vettore AL, Ramnarayanan K, Poore G, Lim K, Ong CK, Huang KK, Leong HS, Chong FT, Lim TK, Lim WK, et al: Mutational landscapes of tongue carcinoma reveal recurrent mutations in genes of therapeutic and prognostic relevance. Genome Med 7: 98, 2015

9. India Project Team of the International Cancer Genome Consortium: Mutational landscape of gingivo-buccal oral squamous cell carcinoma reveals new recurrently-mutated genes and molecular subgroups. Nat Commun 4: 2873, 2013.

10. Ojesina AI, Lichtenstein L, Freeman SS, Pedamallu CS Imaz-Rosshandler I, Pugh TJ, Cherniack AD, Ambrogio L, Cibulskis K, Bertelsen B, et al: Landscape of genomic alterations in cervical carcinomas. Nature 506: 371-375, 2014.

11. Ranganathan P, Weaver KL and Capobianco AJ: Notch signalling in solid tumours: A little bit of everything but not all the time. Nat Rev Cancer 11: 338-351, 2011.

12. Vicente C, Schwab C, Broux M, Geerdens E, Degryse S, Demeyer S, Lahortiga I, Elliott A, Chilton L, La Starza R, et al: Targeted sequencing identifies associations between IL7R-JAK mutations and epigenetic modulators in T-cell acute lymphoblastic leukemia. Haematologica 100: 1301-1310, 2015.
13. Radtke F and Raj K: The role of Notch in tumorigenesis: Oncogene or tumour suppressor? Nat Rev Cancer 3: 756-767, 2003.

14. Leong KG and Karsan A: Recent insights into the role of Notch signaling in tumorigenesis. Blood 107: 2223-2233, 2006.

15. Yap LF, Lee D, Khairuddin A, Pairan MF, Puspita B, Siar CH and Paterson IC: The opposing roles of NOTCH signalling in head and neck cancer: A mini review. Oral Dis 21: 850-857, 2015.

16. Aoyama K, Ota Y, Kajiwara K, Hirayama N and Kimura M: Frequent mutations in NOTCH1 ligand-binding regions in Japanese oral squamous cell carcinoma. Biochem Biophys Res Commun 452: 980-985, 2014.

17. Mizutani A, Kikkawa E, Matsuno A, Shigenari A, Okinaga H, Murakami M, Ishida H, Tanaka $M$ and Inoko $H$ : Modified S/MAR episomal vectors for stably expressing fluorescent protein-tagged transgenes with small cell-to-cell fluctuations. Anal Biochem 443: 113-116, 2013.

18. Agrawal N, Frederick MJ, Pickering CR, Bettegowda C, Chang K, Li RJ, Fakhry C, Xie TX, Zhang J, Wang J, et al: Exome sequencing of head and neck squamous cell carcinoma reveals inactivating mutations in NOTCH1. Science 333: 1154-1157, 2011.

19. Sun Y, Zhang R, Zhou S and Ji Y: Overexpression of Notch1 is associated with the progression of cervical cancer. Oncol Lett 9: 2750-2756, 2015 .

20. Su BH, Qu J, Song M, Huang XY, Hu XM, Xie J, Zhao Y, Ding LC, She L, Chen J, et al: NOTCH1 signaling contributes to cell growth, anti-apoptosis and metastasis in salivary adenoid cystic carcinoma. Oncotarget 5: 6885-6895, 2014.

21. Wang Y, Yu S, Huang D, Cui M, Hu H, Zhang L, Wang W, Parameswaran N, Jackson M, Osborne B, et al: Cellular prion protein mediates pancreatic cancer cell survival and invasion through association with and enhanced signaling of Notch1. Am J Pathol 186: 2945-2956, 2016.

22. Hsu KW, Fang WL, Huang KH, Huang TT, Lee HC, Hsieh RH, Chi CW and Yeh TS: Notch1 pathway-mediated microRNA151-5p promotes gastric cancer progression. Oncotarget 7 : 38036-38051, 2016.

23. Croquelois A, Domenighetti AA, Nemir M, Lepore M, Rosenblatt-Velin N, Radtke F and Pedrazzini T: Control of the adaptive response of the heart to stress via the Notch1 receptor pathway. J Exp Med 205: 3173-3185, 2008.

24. Pickering CR, Zhang J, Yoo SY, Bengtsson L, Moorthy S, Neskey DM, Zhao M, Ortega Alves MV, Chang K, Drummond J, et al: Integrative genomic characterization of oral squamous cell carcinoma identifies frequent somatic drivers. Cancer Discov 3: 770-781, 2013.

25. Shao S, Zhao X, Zhang X, Luo M, Zuo X, Huang S, Wang Y, $\mathrm{Gu} S$ and Zhao X: Notch1 signaling regulates the epithelialmesenchymal transition and invasion of breast cancer in a Slug-dependent manner. Mol Cancer 14: 28, 2015.

26. Ma L, Dong P, Liu L, Gao Q, Duan M, Zhang S, Chen S, Xue R and Wang X: Overexpression of protein O-fucosyltransferase 1 accelerates hepatocellular carcinoma progression via the Notch signaling pathway. Biochem Biophys Res Commun 473: 503-510, 2016.

27. Okajima T, Xu A, Lei L and Irvine KD: Chaperone activity of protein $\mathrm{O}$-fucosyltransferase 1 promotes notch receptor folding. Science 307: 1599-1603, 2005.

28. Yoshida R, Nagata M, Nakayama H, Niimori-Kita K, Hassan W, Tanaka T, Shinohara M and Ito T: The pathological significance of Notch1 in oral squamous cell carcinoma. Lab Invest 93: 1068-1081, 2013.

29. Sun W, Gaykalova DA, Ochs MF, Mambo E, Arnaoutakis D, Liu Y, Loyo M, Agrawal N, Howard J, Li R, et al: Activation of the NOTCH pathway in head and neck cancer. Cancer Res 74: 1091-1104, 2014.

30. Jung J: Human tumor xenograft models for preclinical assessment of anticancer drug development. Toxicol Res 30: 1-5, 2014.

31. Weber JM and Calvi LM: Notch signaling and the bone marrow hematopoietic stem cell niche. Bone 46: 281-285, 2010. 